\title{
Enhanced electron spin rotation in CdS quantum dots
}

\author{
Yasuaki Masumoto*, Hikaru Umino, Jianhui Sun, and Eri Suzumura
}

\author{
Received Xth $X X X X X X X X X X 20 X X$, Accepted Xth $X X X X X X X X X 20 X X$ \\ First published on the web Xth $X X X X X X X X X X 200 X$ \\ DOI: 10.1039/b000000x
}

\begin{abstract}
We studied the spin rotation of electrons in CdS quantum dots (QDs) and CdS QDs with charge acceptors by means of timeresolved Faraday rotation (TRFR) at room temperature. The electron spin rotation gave an oscillatory component in TRFR signal and the oscillation frequency proportional to magnetic field gave the $g$-factor of electrons $1.965 \pm 0.006$. Non-oscillatory component comes from population of excitons and showed additional decay in CdS QDs with hole acceptor. Electron spin rotation signal was largely enhanced and lasted for spin coherence time of $T_{2}^{*}=450 \mathrm{ps}$ in $\mathrm{CdS}$ QDs tethered to TiO $\mathrm{O}_{2}$ electron acceptor, where the spin initialization was triggered by the positive trion transition. These results give clear evidence that the electron spin rotation signal in QDs can be enhanced by transient $p$-doping.
\end{abstract}

\section{Introduction}

Spins of both localized electrons in semiconductors and confined electrons in semiconductor quantum dots (QDs) have long coherence time. They are candidates of solid-state qubits for quantum information processing. It is desirable for electron spins to be coherent for long time at room temperature. Time-resolved Faraday rotation (TRFR) was used to measure the excitonic population and spin rotation of electrons in $\mathrm{CdSe}$ and CdS QDs under the transverse magnetic field at room temperature $^{1,2}$. Low natural abundance of nuclear spins and small hyperfine constant of $\mathrm{Cd}$ make spin coherence time of electrons in CdSe and CdS QDs long. Gupta et al. considered spins of photoexcited electrons are rotating in CdSe QDs ${ }^{1}$. On the other hand, Feng et al. considered spins of residual electrons are rotating in nominally undoped CdS QDs and that residual electrons come from oleic acid capping the surface of QDs ${ }^{2}$. But, initialization mechanism of electron spins is not clear. To clarify the electron source and spin initialization mechanism, TRFR study of intentionally doped QDs is favorable.

So far chemically grown QDs were intentionally doped by the formation of the shell in the type-II alignment. In photoexcited type-II CdTe/CdSe core-shell QDs, He et al. uniquely reported that electron-hole separation in QDs suppressed the electron-hole exchange interaction and lengthened the spin relaxation time of holes ${ }^{3}$. But valid comparison of type-II $\mathrm{CdTe} / \mathrm{CdSe}$ core-shell QDs with type-I CdTe/CdSe core-shell QDs and bare CdTe QDs requires the same growth process of three kinds of samples. Comparison of p-type QDs with undoped QDs grown by the same process is preferable.

Efficient acceptor of electrons are known by the study of

Institute of Physics, University of Tsukuba, Tsukuba 305-8571, Japan E-mail: masumoto@physics.px.tsukuba.ac.jp; Tel: +81-29-853-4248. the QDs-sensitized solar cells of the Grätzel type. Fast and efficient electron transfer from QDs to the porous $\mathrm{TiO}_{2}$ film was observed by means of time-resolved pump-probe spectroscopy and time-correlated single photon counting of luminescence ${ }^{4-6}$, when QDs are tethered to $\mathrm{TiO}_{2}$ through molecular linkers. Hole acceptor molecules for QDs are known, too $^{7,8}$. By assembling QDs donor and acceptor of electrons (holes), we can extract electrons (holes) from photo-excited QDs and have transient $p$-type (n-type) QDs, assuming the remained holes (electrons) have long lifetime comparable to the repetition period of the excitation pulses. We can obtain undoped, p-type and n-type QDs from the same QDs stock solution.

Electrons, holes and excitons in semiconductor have different $g$-factors and different spin rotation frequencies in the magnetic field. In this work, we observed electron spin rotation in free-standing CdS QDs, CdS QDs tethered to electron acceptor and CdS QDs surrounded by hole acceptor by means of TRFR. Electron spin rotation in CdS QDs tethered to electron acceptor was prolonged and highly enhanced. Excitonic population and spin rotation of electrons showed additional dumping in CdS QDs surrounded by hole acceptor.

\section{Experimental}

\subsection{Synthesis of colloidal CdS quantum dots}

The colloidal CdS QDs were synthesized following the method reported by Peng et al. ${ }^{9}$. First, sulfide precursor was prepared by mixing $38.4 \mathrm{mg}$ sulfur powder and $6 \mathrm{ml}$ of 1 octadecene (ODE). The mixture were heated to $60{ }^{\circ} \mathrm{C}$ under stirring. Then, a mixture of $307.2 \mathrm{mg}$ cadmium oxide $(\mathrm{CdO})$, $2.24 \mathrm{ml}$ oleic acid (OA), and $28.56 \mathrm{ml}$ ODE were loaded in the three-necked flask. The mixture was degassed and then 
was heated at the temperature of $100{ }^{\circ} \mathrm{C}$ for 1 hour. After that, the flask was purged with argon and was heated up to the temperature of $270{ }^{\circ} \mathrm{C}$. At the temperature of $270{ }^{\circ} \mathrm{C}$, the prepared sulfide precursor was swiftly injected into the threenecked flask for the growth of CdS QDs. After the growth, the flask was cooled down to room temperature. The CdS QDs solution was loaded in the centrifuge tubes with excess amount of acetone and was centrifuged at rotation speed of $8000 \mathrm{rp}$ $\mathrm{m}$. The yellow solid was redissolved in hexane. A few $\mathrm{ml}$ of acetone was dropwise into the solution and the solution was then centrifuged at a rotation speed of at 2000-4000 rpm. The left yellow CdS QDs solids were redissolved in toluene for storage.

\subsection{CdS QD-MPA-TiO 2 and CdS QD-TPD suspensions for spectroscopic measurements}

Colloidal amorphous $\mathrm{TiO}_{2}$ particles $20 \mathrm{~nm}$ in diameter were made from titanium isopropoxide in the same way as reported in the reference ${ }^{4,11}$. By adding solution of 3mercaptopropionic acid (MPA, HS- $\left.\left(\mathrm{CH}_{2}\right)_{2}-\mathrm{COOH}\right)$ to suspension of $\mathrm{TiO}_{2}$ particles, MPA-capped $\mathrm{TiO}_{2}$ particles having the thiol group outside are made as reported in the reference ${ }^{5}$. Further, functionalized $\mathrm{TiO}_{2}$ particles were mixed with $\mathrm{CdS}$ QDs in toluene. As a result, CdS QDs are tethered to $\mathrm{TiO}_{2}$ particles through the thiol group and the carboxy group of linker molecules MPA, and linked CdS QDMPA-TiO 2 suspension was obtained. Suspension of CdS QD-TPD was obtained by adding N,N'-Bis(3-methylphenyl)$\mathrm{N}, \mathrm{N}$ '-bis(phenyl)benzidine (TPD) to $\mathrm{CdS}$ QDs solution in toluene.

\subsection{Time-resolved Faraday rotation spectroscopy}

Three kinds of samples, CdS QD, CdS QD-MPA-TiO 2 and CdS QD-TPD, in cuvettes $1 \mathrm{~mm}$ thick were placed at the center of pole pieces of the electromagnet for the TRFR measurement at room temperature. The $225 \mathrm{fs}$ laser pulses were generated every $4 \mu$ s by a $250 \mathrm{kHz}$ Ti:sapphire regenerative amplifier. The center wavelength and the band width is $800 \mathrm{~nm}$ and $20 \mathrm{~nm}$, respectively. Their second harmonic pulses covering the absorption edge of the CdS QDs were used as pump and probe pulses. The polarization of pump pulses was modulated by a $41 \mathrm{kHz}$ photoelastic modulator (PEM) and was changed to right or left circular polarization alternatively. The linear polarization axis of the probe was rotated by 45 degrees by a half-wave plate. These pump and probe beams were focused on the sample in the transverse magnetic field geometry. The probe pulses passing through the sample were divided into two cross-linearly polarized components by a Rochon prism and two components were detected by balanced photodiodes. The differential signal from the detector was amplified by a lock-in amplifier at the the modulation frequency of PEM and its output was amplified again by the second lock-in amplifier at the mechanical chopping frequency of $210 \mathrm{~Hz}$.

\section{Results and discussion}

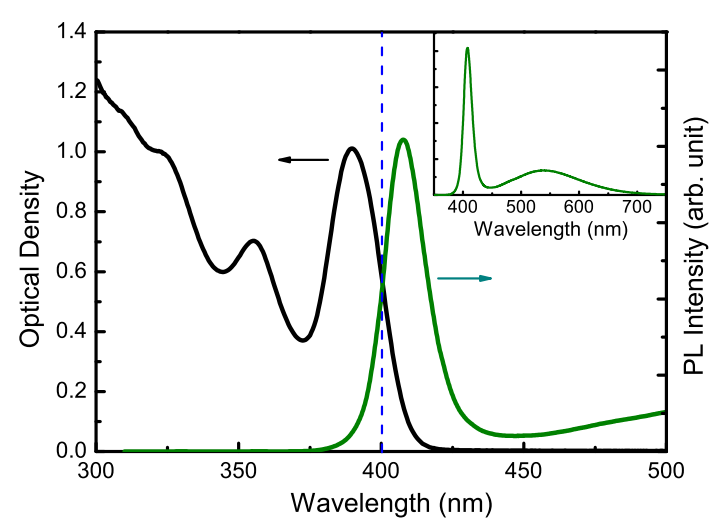

Fig. 1 Absorption and photoluminescence (PL) spectra of CdS QDs $3.0 \mathrm{~nm}$ in average diameter in toluene shown by a black line and a green line, respectively. The inset shows the PL spectrum extending to longer wavelength. The pump and probe photon energies in the time-resolved Faraday rotation experiment are degenerate and are shown by a vertical blue dashed line.

The absorption and photoluminescence (PL) spectrum of CdS QDs in toluene are shown in Fig.1. The lowest energy peak in the absorption spectrum is located at $389 \mathrm{~nm}$ which gives the average diameter of $3.0 \mathrm{~nm}$ based on the relationship between the diameter of QDs and the absorption peak wavelength in the bibliography ${ }^{10}$. Photoluminescence spectrum peaks at $408 \mathrm{~nm}$ and the Stokes shift, $19 \mathrm{~nm}$, suggests the exciton localization in QDs. A broad luminescence spectrum peaked at $540 \mathrm{~nm}$ comes from recombination between electrons and holes trapped separately at the surface of $\mathrm{CdS}$ QDs ${ }^{11}$. Time-correlated single photon counting measurement of CdS QDs showed the excitonic luminescence decays multiexponentially and tails off at $3 \mu \mathrm{s}$. Luminescence around 540 $\mathrm{nm}$ decays multiexponentially and tails off at $5 \mu \mathrm{s}$. Electronhole separated state is kept for long time.

The lowest unoccupied molecular orbital (LUMO) level$\mathrm{s}$ and the highest occupied molecular orbital (HOMO) levels of CdS QDs $3.0 \mathrm{~nm}$ in diameter, amorphous $\mathrm{TiO}_{2}$ particles and TPD molecules are shown in Fig.2. Cyclic voltammetry and optical absorption measurements gave CdS QDs 3.0 $\mathrm{nm}$ in diameter the electron affinity of $-3.35 \mathrm{eV}$ and ionization potential of $-6.6 \mathrm{eV}$. They gave amorphous $\mathrm{TiO}_{2}$ particles 


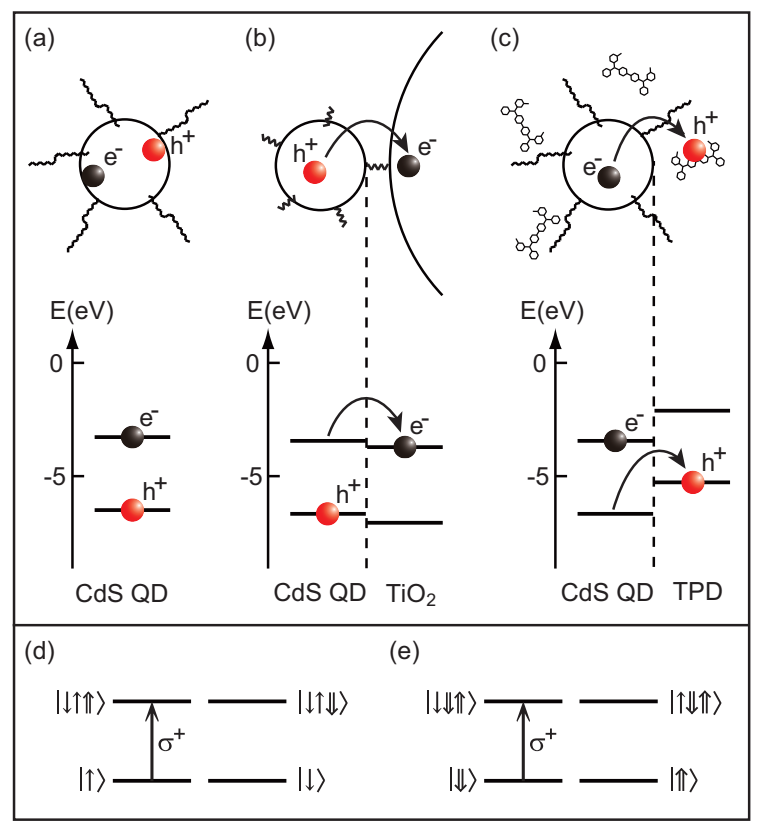

Fig. 2 Top row: Drawings of (a) a CdS QD partially capped by oleic acid, (b) a CdS QD tethered to a $\mathrm{TiO}_{2}$ nanoparticle through 3mercaptopropionic acid (MPA), and (c) a CdS QD partially capped by oleic acid with TPD molecules. Middle row: Schematic energy level diagram of the LUMO and HOMO levels of CdS QDs, amorphous $\mathrm{TiO}_{2}$ and TPD. Possible electron (hole) transfer from QDs donor to $\mathrm{TiO}_{2}$ (TPD) acceptor is shown by an arrow. Bottom row: Spin initialization by the trion transition. Electron spin is denoted by $|\downarrow\rangle$ or $|\uparrow\rangle$, and hole spin is denoted by $|\downarrow\rangle$ or $|\uparrow\rangle$. Right circular polarization is denoted by $\sigma^{+}$.

the electron affinity of $-3.95 \mathrm{eV}$ and ionization potential of $7.15 \mathrm{eV}$. It is known that TPD has the electron affinity of -2.1 $\mathrm{eV}$ and ionization potential of $-5.4 \mathrm{eV}^{7}$. We can expect electron transfer from CdS QDs to $\mathrm{TiO}_{2}$ particles and hole transfer from CdS QDs to TPD, as schematically shown in Fig.2. Excitonic luminescence quenching was observed in CdS QDMPA-TiO was observed in CdS QD-TPD. Luminescence quenching was observed around $540 \mathrm{~nm}$ in CdS QD-MPA-TiO 2 , too, as was reported by the reference ${ }^{11}$. Reflecting electron transfer from CdS QDs donor to $\mathrm{TiO}_{2}$ acceptor, electron-hole separated state is preserved for long time. Its lifetime was measured to be $2.6 \mu$ s by transient absorption ${ }^{11}$.

The TRFR signals of three kinds of samples have the different amplitudes of the oscillatory component and the nonoscillatory component, as shown in Fig.3. Note that the vertical scale interval means the same intensity of the TRFR signal. The TRFR signal of CdS QD-MPA-TiO 2 has largely enhanced oscillatory component and that of CdS QD-TPD has decaying non-oscillatory component. All the TRFR sig-

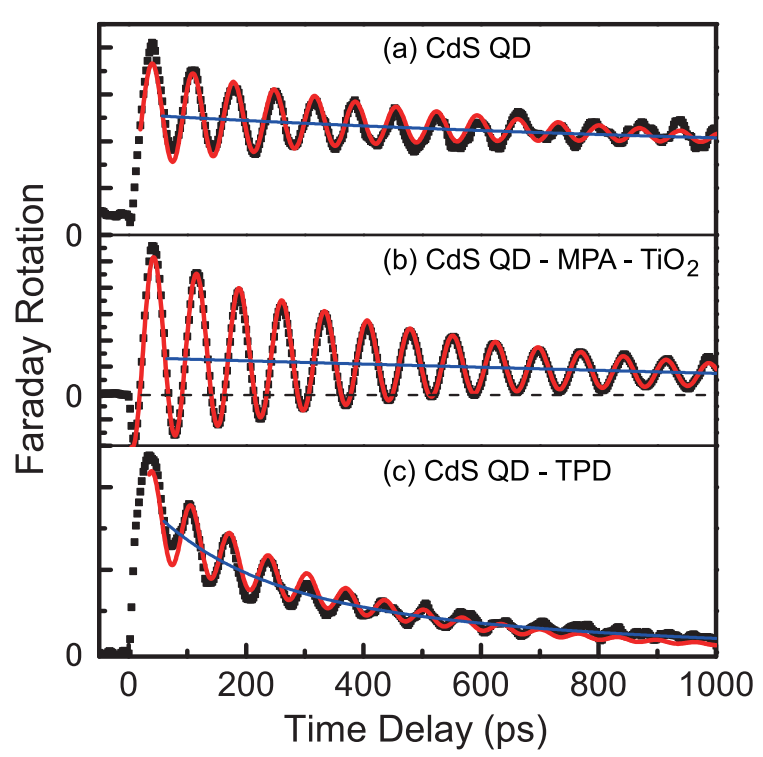

Fig. 3 Time-resolved Faraday rotation (TRFR) signal (solid squares) for (a) $\mathrm{CdS} \mathrm{QD}$ under $B=0.539 \mathrm{~T}$, (b) $\mathrm{Cd}$ $\mathrm{S}$ QDs-MPA- $\mathrm{TiO}_{2}$ under $B=0.528 \mathrm{~T}$ and (c) $\mathrm{CdS}$ QDsTPD under $B=0.539$ T. Red curves are fitting by (a) $5.20 \exp (-t / 4510[\mathrm{ps}])+2.40 \exp (-t / 404[\mathrm{ps}]) \cos (2 \pi t / 69.1[\mathrm{ps}]-$

$0.67 \pi), \quad$ (b) $\quad 3.45 \exp (-t / 1110[\mathrm{ps}])+7.69 \exp (-t / 450[\mathrm{ps}])$ $\cos (2 \pi t / 72.7[\mathrm{ps}]-0.68 \pi)$ and (c) $3.75 \exp (-t / 341[\mathrm{ps}])+$ $1.22 \exp (-t / 257[\mathrm{ps}]) \cos (2 \pi t / 66.1[\mathrm{ps}]-0.73 \pi)$ and blue curves represent the nonoscillatory component.

nals are written in common by the sum of the dumped oscillation coming from Larmor precession of the carrier spins and the single exponential decay. The time constant of the exponential decay means the dephasing time of the spin ensemble. The TRFR signal $\theta_{F}(t)$ was fitted by the equation, $\theta_{F}(t)=a_{1} \exp (-t / \tau)+a_{2} \exp \left(-t / T_{2}^{*}\right) \cos \left(2 \pi v_{L} t+\phi\right)$, where $a_{1}, a_{2}, \tau, T_{2}^{*}, v_{L}$ and $\phi$ are fitting parameters. Parameters $a_{1}, \tau$ and $a_{2}$ denote an amplitude of the nonoscillatory component, a decay constant of the nonoscillatory component and an amplitude of the oscillatory component, respectively. Parameters $v_{L}$ and $T_{2}^{*}$ correspond to the Larmor frequency and the dephasing time of the spin ensemble, respectively. A parameter $\phi$ is the initial phase. Comparing the fitting parameters, we note $T_{2}^{*}$ is longest for CdS QD-MPA-TiO 2 and that $T_{2}^{*}$ is shortest for Cd$\mathrm{S}$ QD-TPD. The amplitude of oscillatory component is largest for CdS QD-MPA-TiO 2 and smallest for CdS QD-TPD.

Magnetic field dependence of the Larmor frequency $v_{L}$ gives the $g$-factor, $g=h v_{L} / \mu_{B} B$, where $\mu_{B}$ is the Bohr magneton and $B$ is the magnetic field. As is shown in Figs.4(a) and $4(\mathrm{c}), v_{L}$ is propotional to $B$ for CdS QDs-MPA-TiO ${ }_{2}$, and the slope gives the $g$-factor. The similar linear dependence held for CdS QD and CdS QD-TPD. The $g$-factors obtained are $g=1.965 \pm 0.006,1.95 \pm 0.05$ and $1.91 \pm 0.06$ for CdS QD, 

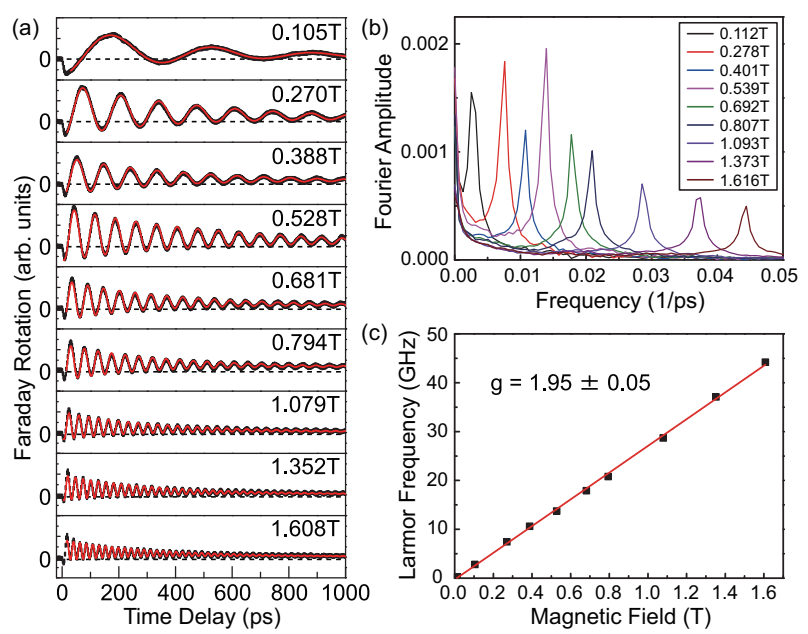

Fig. 4 (a) The TRFR signals (solid squares) in CdS QD-MPA-TiO 2 under transverse magnetic field. Red curves are fittings by a single exponential decay plus a dumped oscillation. (b) Fourier transform of the TRFR signals. (c) Larmor frequency (solid squares) as a function of transverse magnetic field. A red linear fitting gives the $g$-factor of $1.95 \pm 0.05$.

CdS QD-MPA-TiO 2 and CdS QD-TPD, respectively. These $g$-factors agree with each other within the margin of errors. Therefore we took $g=1.965 \pm 0.006$ having the smallest error. Fourier transform of the TRFR signals shown in Fig.4(b) consists of a single frequency peak. Thus the oscillation signal comes from one of an electron, a hole and an exciton.

In hexagonal $\mathrm{CdS}$ crystal having $c$-axis, $g_{e \perp}=1.72 \pm 0.1$ and $g_{e \|}=1.78 \pm 0.05$ are known ${ }^{12}$, where $g_{e \perp}$ and $g_{e \|}$ are electron $g$-factors for $B \perp c$ and $B \| c$, respectively. Therefore $g$-factor of electron in $\mathrm{CdS}$ crystal is isotropic within the experimental error and is denoted by $g_{e}$. On the other hand, the $g$-factor of hole in hexagonal CdS crystal are highly anisotropic and $g_{h \perp}=0$ and $g_{h \|}=1.15 \pm 0.05$ are known ${ }^{12,13}$. When the angle between $B$ and the $c$-axis is $\theta$, the $g$-factor of hole is given by $g_{h \|} \cos \theta^{13}$. The bright A-exciton $g$-factor is given by $g_{e}$, $g_{e}-g_{h \|}$ and $g_{e}-g_{h \|} \cos \theta$ for $B \perp c, B \| c$ and $\angle(B, c)=\theta$, respectively. If the $c$-axis is oriented randomly to the magnetic field like CdS QDs colloids, the $g$-factors of hole and exciton are distributed from 0 to 1.15 and from $g_{e}-1.15$ to $g_{e}$, respectively. In this case, we can not observe long-lasting oscillatory signal of the Faraday rotation of spins for holes and excitons in hexagonal CdS QDs, but can observe fast dumping of oscillatory signal within the inverse of broadening of Larmor freuency, $2 \pi \hbar / 1.15 \mu_{B} B$. At $B=0.5 \mathrm{~T}$, it is $124 \mathrm{ps}$. We can exclude spin rotation of holes and excitons to explain the oscillatory component in the TRFR signal.

Because the electron $g$-factor alone is isotropic, the electron spin rotation in CdS QDs gives the long-lasting oscilla- tory component in the TRFR signal. The electron $g$-factor in QDs is evaluated by the modified Roth formula, $g=g_{0}-$ $(2 / 3) E_{P} \Delta_{s o} /\left[\left(E_{g}+E_{c}+\Delta_{s o}\right)\left(E_{g}+E_{c}\right)\right]$, where $g_{0}=2$ is the $g$-factor of the free electron, $E_{P}$ is the Kane energy parameter, $\Delta_{s o}$ is the spin-orbit splitting of the valence band, $E_{g}$ is the band gap energy of the bulk semiconductor, and $E_{c}$ is the electron confinement energy ${ }^{14}$. Therefore the electron $g$-factor in CdS QDs is considered to approarch to $g_{0}=2$ with the decrease in size, because of the confinement-induced energy shift of the electron energy in the conduction band. Using the CdS parameters, $E_{g}=2.40 \mathrm{eV}, E_{P}=21.0 \mathrm{eV}$ and $\Delta_{s o}=0.062 \mathrm{eV}$, ${ }^{15}$ and the CdS QD parameter, $E_{g}+E_{c} \sim 3.185 \mathrm{eV}$, we can evaluate the confinement-induced increase of the electron $g$-factor to be 0.063 . The estimate is smaller than the measured increase of 0.2 , but the $g$-factor of $1.965 \pm 0.006$ is regarded as the $g$ factor of electron in CdS QDs $3.0 \mathrm{~nm}$ in diameter. Similar electron $g$-factor of 1.93 was reported for CdS QDs $5.6 \mathrm{~nm}$ in diameter $^{2}$.

Circularly polarized laser pulse excites population of excitons in CdS QDs having no residual electrons and holes and produces the nonoscillatory component in the TRFR signal ${ }^{2}$. However, considerable portion of both electrons and holes remains in CdS QDs at $4 \mu$ s after the preceding pulses excite the electron-hole pairs, because lifetime of electron-hole separated state was as long as $2.6 \mu \mathrm{s}^{11}$. In the transverse magnetic field, electron spin rotation starts after right (left) circularly polarized laser pulse excites negative trion composed of a hole and two spin-singlet electrons $|\downarrow \uparrow \downarrow\rangle(|\downarrow \uparrow \uparrow\rangle)$ from a residual electron $|\downarrow\rangle(|\uparrow\rangle)$ as is shown in Fig.2(d), because electron spin population at the $|\uparrow\rangle(|\downarrow\rangle)$ state becomes larger than that at the $|\downarrow\rangle(|\uparrow\rangle)$ state $^{16}$. From a residual hole $|\Downarrow\rangle(|\Uparrow\rangle)$, right (left) circularly polarized laser pulse excites the positive trion composed of two spin-singlet holes and an electron $|\downarrow \Uparrow \downarrow\rangle$ $(|\uparrow \uparrow \Downarrow\rangle)$ as is shown in Fig.2(e), and positive trion spin, that is equivalent to electron spin, starts rotating in the transverse magnetic field.

In CdS QD-MPA-TiO 2 composite, electron transfer from QDs to $\mathrm{TiO}_{2}$ takes place efficiently at the time constant of 1110 ps in consistent with electron transfer rate reported for CdSe QD-MPA-TiO 2 having the similar LUMO energy difference, $0.6 \mathrm{eV}$, between donor and acceptor ${ }^{4}$. Reflecting the transfer time of electrons, non-oscillatry component coming from population of excitons decays at the time constant of $1110 \mathrm{ps}$. Then considerable portion of holes remains in QDs at $4 \mu \mathrm{s}$ after the preceding pulses excites the trion composed of an electron and 2 holes, because lifetime of electron-hole separated state was as long as $2.6 \mu \mathrm{s}^{11}$. The positive trion transition from a residual hole shown in Fig.2(e) initializes the spin rotation of electron. Transient $p$-doping of QDs increased the spin rotation signal of electrons by 3 times and clarified spin initialization mechanism of the positive trion transition. In CdS QD-TPD, photoexcited holes are transfered from QDs to 
TPD and electrons are remained in QDs. The spin initialization by the negative trion transition shown in Fig.2(d) works in CdS QD-TPD. Population of excitons decays at the time constant of 341 ps reflecting the transfer rate of holes.

The dephasing relaxation time of $T_{2}^{*}$ is $400 \mathrm{ps}, 450 \mathrm{ps}$ and 260 ps in CdS QD, CdS QD-MPA-TiO 2 and CdS QD-TPD, respectively. The longest $T_{2}^{*}$ was observed in CdS QD-MPA$\mathrm{TiO}_{2}$. This means electron spin relaxation is slowest in $p$ doped QDs, where a positive trion spin alone is rotating in QDs after the photoexcitation. Electron transfer time from QDs to $\mathrm{TiO}_{2}, 1110 \mathrm{ps}$, is longer than $T_{2}^{*}$ of electron spin dephasing time, $450 \mathrm{ps}$, and therefore reduces $T_{2}^{*}$ little. Low natural abundance of nuclear spins $\left[{ }^{111} \mathrm{Cd}(I=1 / 2,12.80 \%)\right.$, $\left.{ }^{113} \mathrm{Cd}(I=1 / 2,12.22 \%),{ }^{33} \mathrm{~S}(I=3 / 2,0.75 \%)\right]$ and small hyperfine constant of $\mathrm{Cd}(I=1 / 2)$ in CdSe and CdS QDs make spin coherence time of electrons as long as $T_{2}^{*}=450 \mathrm{ps}$ at room temperature.

\section{Conclusions}

In conclusion, we applied TRFR measurement to CdS QDs and CdS QDs with charge acceptors, $\mathrm{TiO}_{2}$ nanoparticles and TPD molecules, at room temperature. We found that TRFR signal is the sum of the oscillatory signal coming from the rotation of electron spins and non-oscillatory signal coming from the excitonic population in CdS QDs. The $g$-factor was determined to be $g=1.965 \pm 0.006$ from the oscillation frequency proportional to magnetic field, and the dephasing relaxation time of $T_{2}^{*}$ was about $450 \mathrm{ps}$ for CdS QDs tethered to $\mathrm{TiO}_{2}$. Non-oscillatory component comes from population of excitons and showed additional decay in CdS QDs with hole acceptor. Electron spin rotation signal is largely enhanced in CdS QDs tethered to $\mathrm{TiO}_{2}$. The spin initialization was triggered by the positive trion transition. Transient $p$-doping of QDs increased the electron spin rotation signal.

\section{Acknowledgement}

This work was supported by the Grant-in-Aid for the Scientific Research $\sharp 26400309$ from the Ministry of Education, Sports, Culture, Science and Technology of Japan.

\section{References}

1 Gupta, J. A.; Awschalom, D. D.; Efros, Al. L.; Rodina, A. V. Phys. Rev. B 2002, 66, 125307.

2 Feng, D. H.; Li, X.; Jia, T. Q.; Pan, X. Q.; Sun, Z. R. and Xu, Z. Z. Appl. Phys. Lett. 2012, 100, 122406.

3 He, J.; Zhong, H. and Scholes, G. D. Phys. Rev. Lett. 2010, 105, 046601 .
4 Robel, I.; Subramanian, V.; Kuno, M. and Kamat, P. V. J. Am. Chem. Soc. 2006, 128, 2385.

5 Hyun, B.-R.; Zhong, Yu-Wu.; Bartnik, A. C.; Sun, L.; Abruña, H. D.; Wise, F. W.; Goodreau, J. D.; Matthews, J. R.; Leslie, T. M. and Borrelli, N. F. ACS Nano 2008, 2, 2206.

6 Masumoto, Y.; Takagi, H.; Umino, H. and Suzumura, E. F. Appl. Phys. Lett. 2012, 100, 252106.

7 Coe, S.; Woo, W.-K.; Bawendi, M. and Bulović, V. Nature 2002, 420, 800.

8 Jing, P.; Yuan, X.; Ji, W.; Ikezawa, M.; Liu, X.; Zhang, L.; Zhao, J. and Masumoto, Y. Appl. Phys. Lett. 2011, 99 , 093106.

9 Yu, W. W. and Peng, X. Angew. Chem. Int. Ed. 2002, 41, 2368.

10 Yu, W. W.; Qu, L.; Guo, W. and Peng, X. Chem. Mater. 2003, 15, 2854.

11 Dibbell, R. S. and Watson, D. F. J. Phys. Chem. C. 2009, 113, 3139.

12 Hopfield, J. J. and Thomas, D. G. Phys. Rev. 1961, 122, 35.

13 Thomas, D. G. and Hopfield, J. J. Phys. Rev. 1962, 128, 2135.

14 Kiselev, A.A.; Ivchenko, E. L.; Rössler, U. Phys. Rev. B 1998, 58, 16353.

15 Ithurria, S.; Tessier, M. D.; Mahler, B.; Lobo, R. P. S. M.; Dubertret, B. and Efros, Al. L. Nat. Mater. 2011, 10, 936.

16 Greilich, A.; Oulton, R.; Zhukov, E. A.; Yugova, I. A.; Yakovlev, D. R.; Bayer, M.; Shabaev, A.; Efros, Al. L.; Merkulov, I. A.; Stavarache, V.; Reuter, D. and Wieck, A. Phys. Rev. Lett. 2006, 96, 227401. 